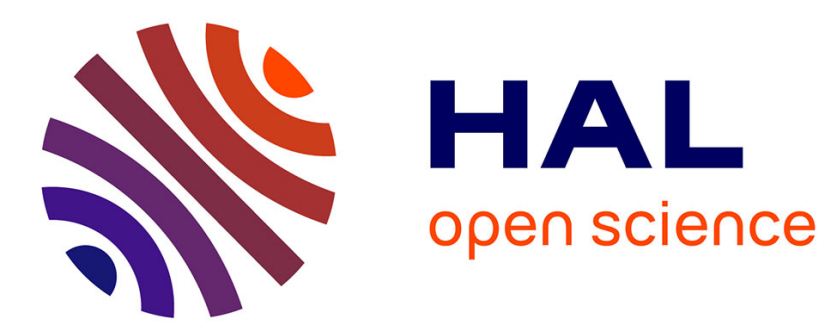

\title{
Dynamic voltage scaling for series hybrid amplifiers
}

Patrice Russo, Firas Yengui, Gaël Pillonnet, Sophie Taupin, Nacer Abouchi

\section{To cite this version:}

Patrice Russo, Firas Yengui, Gaël Pillonnet, Sophie Taupin, Nacer Abouchi. Dynamic voltage scaling for series hybrid amplifiers. Microelectronics Journal, 2013, pp.11. hal-01103600

\section{HAL Id: hal-01103600 \\ https://hal.science/hal-01103600}

Submitted on 16 Jan 2015

HAL is a multi-disciplinary open access archive for the deposit and dissemination of scientific research documents, whether they are published or not. The documents may come from teaching and research institutions in France or abroad, or from public or private research centers.
L'archive ouverte pluridisciplinaire HAL, est destinée au dépôt et à la diffusion de documents scientifiques de niveau recherche, publiés ou non, émanant des établissements d'enseignement et de recherche français ou étrangers, des laboratoires publics ou privés. 


\section{Elsevier Editorial System(tm) for Microelectronics Journal Manuscript Draft}

Manuscript Number:

Title: Dynamic Voltage Scaling for Series Hybrid Amplifiers

Article Type: Research Paper

Keywords: Audio amplifier; optimization; dynamic voltage scaling; hybrid amplifier; class G/H Corresponding Author: Mr Patrice RUSSO,

Corresponding Author's Institution:

First Author: Patrice RUSSO

Order of Authors: Patrice RUSSO; Gael PILLONNET, Doctor; Firas YENGUI, Msc; Nacer Abouchi, Professor 


\title{
Dynamic Voltage Scaling for Series Hybrid Amplifiers
}

\author{
Patrice RUSSO, Gael PILLONNET, Firas YENGUI and Nacer ABOUCHI \\ University of Lyon, Lyon Institut of Nanotechnology (INL-UMR5270), Lyon, FRANCE \\ patrice.russo@ hotmail.fr, gael.pillonnet@cpe.fr, firas.yengui@gmail.com
}

\begin{abstract}
We present an optimization of the voltage scaling algorithm in low power audio class $\mathrm{G}$ amplifier for headphones application to allow longer playback time. The optimization approach minimizes the voltage difference between the internal audio amplifier power supply and its output signal over a large range of operating conditions. The modeling is based on a behavioral model enabling accurate and rapid evaluation of efficiency and audio quality with realistic input stimuli. The model validated in practice is used to optimize the voltage scaling using only few power supply levels. Thanks to a global search algorithm followed by a local one, the optimization gives the better parameters for voltage scaling algorithm while keeping a good audio quality. The proposed configuration increases the efficiency up to $48 \%$ at nominal operation.
\end{abstract}

\section{Index Terms}

Audio amplifier; optimization; dynamic voltage scaling; hybrid amplifier; class $G / H$ 


\section{INTRODUCTION}

Embedded systems, such as MP3 players, mobile phones, PDAs, and GPSs, integrate more and more complex functions which therefore consume more energy. The playback time of these embedded systems is directly linked to the battery technology used as well as the overall power consumption. Among these functions, the audio part represents about a third of the power consumption in mobile phone (listening music use case). Therefore, the consumption reduction of the integrated audio system is a key factor to improve the embedded system autonomy. The improvement in the energy efficiency of audio amplifiers (for hands-free, ringtone and headphones) and loudspeakers has been the subject of much research work. Our article deals more particularly with the reduction in the power consumption of audio amplifiers for head phones. Due to the functioning time, the headphone application represents one of the main directions for improvement of the playback time in mobile phones.

Since the first generation valve amplifiers enabling audio amplification in 1915 [1], there has been much progress in electronics with the arrival of transistors and associated architectures, such as the linear class$\mathrm{AB}$ amplifier. They have allowed audio signal reproduction with excellent linearity and a relatively low implementation complexity. However, class-AB amplifiers have a limited efficiency in the relation (see (1)) between the RMS voltage of the $\mathrm{V}_{\mathrm{OUT}}$ signal and power supply $\mathrm{V}_{\mathrm{DD}}$ :

$$
\eta_{A B}=\frac{\pi}{4} \times \frac{V_{O U T}}{V_{D D}}
$$

This previous expression is valid for pure sinusoidal signals with a negligible quiescent current considered. In practice, the efficiency is low in real operating conditions. In fact, the sound level required is never the maximum and the audio signals shape are very different from a sinusoidal wave with high dynamic range (with a crest factor between 5 and $20 \mathrm{~dB}$ ). The nominal power delivered to the headphones is therefore less, typically $100 \mu \mathrm{W}$, than the maximum power (about $30 \mathrm{~mW}$ with $32 \Omega$ at $1 \mathrm{Vrms}$ ). The output amplitude is therefore very low compared to the supply voltage leading to an efficiency of only a few percent.

Other structures have been studied in order to improve the efficiency of amplifiers used in headphones. Switched amplifiers, i.e. class-D, [3, 4] show a higher efficiency but with lower and introducing problems of electromagnetic compatibility. In order to combine the advantages of switched architectures (small conduction losses) and linear architectures (small static losses and good linearity), hybrid structures were introduced. Parallel hybrid amplifiers (class-K) $[4,5]$, have a poor efficiency at only $100 \mu \mathrm{W}$ due to their high static consumption.

The series hybrid amplifier, type Class- $\mathrm{G}$ or $\mathrm{H}$, is the architecture offering a considerable increase in nominal efficiency with high linearity. They include a class-AB amplifier supplied by one or two switching 
voltage converters controlled by a level detector (fig. 1). A negative voltage regulator is used to center the common mode of the output signal at zero and thus avoids using an external AC coupling capacitor. The principle is to dynamically supply the linear amplifier as a function of the signal amplitude in order to reduce the conduction losses. The current Class-G topology is shown in fig. 1. In order to improve the efficiency, five blocks can be optimized: the battery cell, the linear amplifier, the speaker, the DC/DC converter and the level detector where the Power Supply Switching Algorithm (PSSA) is implemented. This paper proposes to optimize the level detector by finding the better PSSA without changing the hardware of the other block in realistic operating conditions.

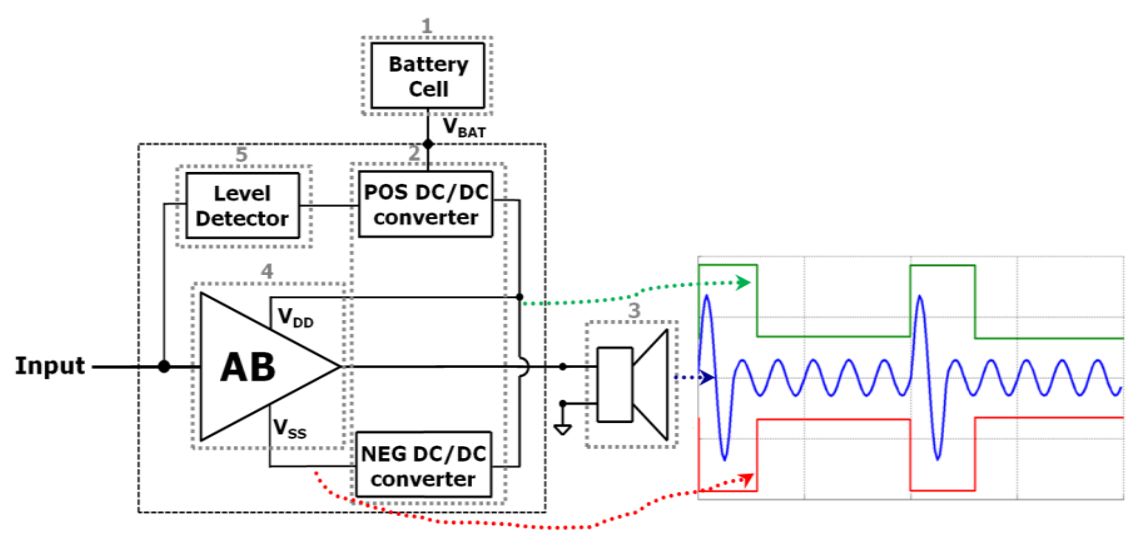

Fig. 1 Class-G block diagram and transient signal

The current Class-G amplifiers use only two or three supply voltages in order to reduce the conduction losses [7]-[9], as illustrated in fig. 1 on the right. Therefore, if the supply voltage is as close as possible to the signal amplitude, then the efficiency of the linear amplifier, given in (1), is maximized. By carefully choosing the number and voltages of the power supplies, the losses due to conduction can be further reduced [10]-[16]. In [11]-[13], the authors suggest an optimization for amplifiers with two or three power supplies, whereas in [14]-[16] they concentrate more on the optimization and implementation of the electrical parameters in order to reduce the consumption and improve the linearity (one of the components of audio quality).

It is important to point out that making the PSSA more complex can result in an increase in the static power consumption. Furthermore, switching the power supplies as close as possible to the signal can, if the variation in signal amplitude is faster than the time required to establish the supply, introduce clipping thus causing deterioration of the audio quality.

The aim of this work is therefore to explore, compare and optimize the PSSA for a hybrid amplifier series in order to reduce power consumption without degrading the quality of sound reproduction. First, a model with reconfigurable switching will be presented to accurately and rapidly simulate the audio quality and power consumption under real operating conditions. Second, the method for optimizing the different 
switching power supplies will be explained. Finally, the validation of the model and its optimization by comparison with an existing circuit, as well as our general approach to different power supply switching strategies will be discussed.

\section{MODELING}

\subsection{Objectives}

With more than 5000 transistors, the present class-G amplifiers [7] require several weeks of calculation to simulate a transient of few milliseconds at transistor level. In order to reduce this calculation time and enable longer transient simulation, a fast and accurate model is suggested. As the simulation time is strictly linked to the level of abstraction, as indicated on fig. 2, behavioral modeling enables a good compromise between time and precision of the simulations [17].

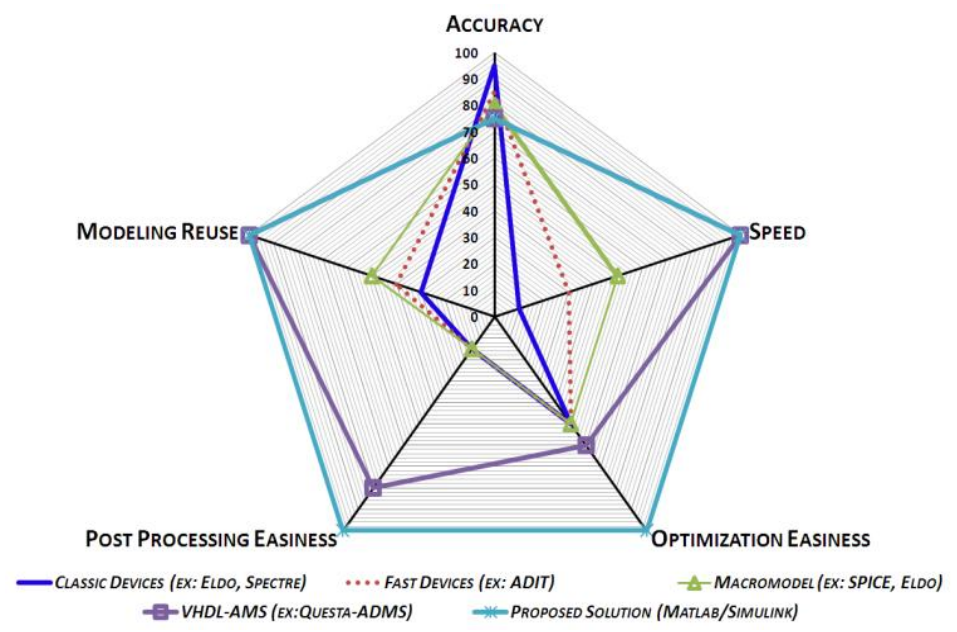

Fig. 2 Modeling comparison

In addition, our model have to allow post-processing to be done on the audio signals (interpolation, and cutting up of sound tracks, etc.), and enable us to evaluate the sound reproduction quality of the audio signal (notably using the PEAQ method). The parameters of our model also need to be optimized via different search algorithms. Finally, the model has to be reconfigurable in order to be able to accept different types of PSSA architecture. We therefore chose a Matlab model, rendering all these actions possible within the same interface.

\subsection{Principle}

The model is based on the present most-used Class-G architecture, shown in fig. 1. It consists of a ClassAB amplifier supplied by two switching DC/DC converters (a DC/DC converter and a charge pump) associated with a level detector to control the power supplies level. The aim of this level detector is to 
switch the power supplies as close as possible to the output signal, without clipping it, in order to reduce the losses in the class- $\mathrm{AB}$ amplifier. It therefore modifies the reference of the two converters as a function of the amplitude of the input signal. However, the amplitude variation of the signal may be faster than the time taken to reach the next power supply value, leading to clipping of the output signal. Furthermore, the present stand-alone class-G amplifiers [7]-[9] with their analog-type inputs do not allow any delay between the decision-making and the amplification of the signal. In order to avoid any eventual clipping, a difference between the output signal amplitude and the power supply is introduced.

We will use the notation Class-Gn for an amplifier with n supply voltages (fig. 3), and Class- $\mathrm{H}$ as an amplifier where the power supply switching is continuous (fig. 6).

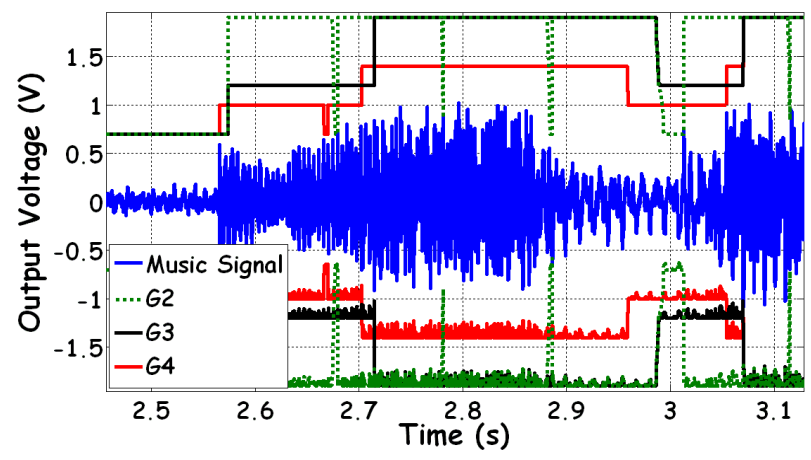

Fig. 3 Class-Gn principle

\subsection{Behavioral modeling}

\subsubsection{PSSA strategies}

The PSSA prameters are completely configurable in the model to be able to determine their influence on power consumption and audio quality. The power supply switching decision is made using the criteria illustrated in fig. 4 and 5 (for the case of class-G2).

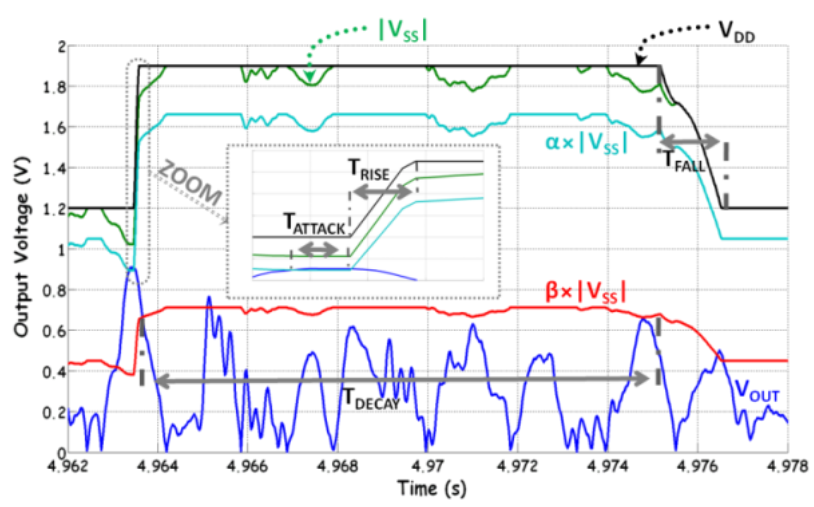

Fig. 4 Gn PSSA parameters 
When the audio signal goes above $\alpha \times\left|V_{S S}\right|$ the power supply value increases to the higher value after a fixed delay called the "attack time". The supply remains constant as long as the signal is not below $\beta \times\left|V_{S S}\right|$. If the latter condition is satisfied for the whole "decay time", then the supply value will decrease to its lower value. The time between these two values $(\operatorname{Vdd} n)$ is defined by the "rise time" and "fall time", respectively. The latter is a function of the discharge time of the output converters capacitor. For the case of a class-G2 amplifier, the PSSA is summarized in fig. 5. When the number of power supplies is greater than two, the "blanking time" parameter is introduced to define a delay between two successive power supplies rises or two successive falls.

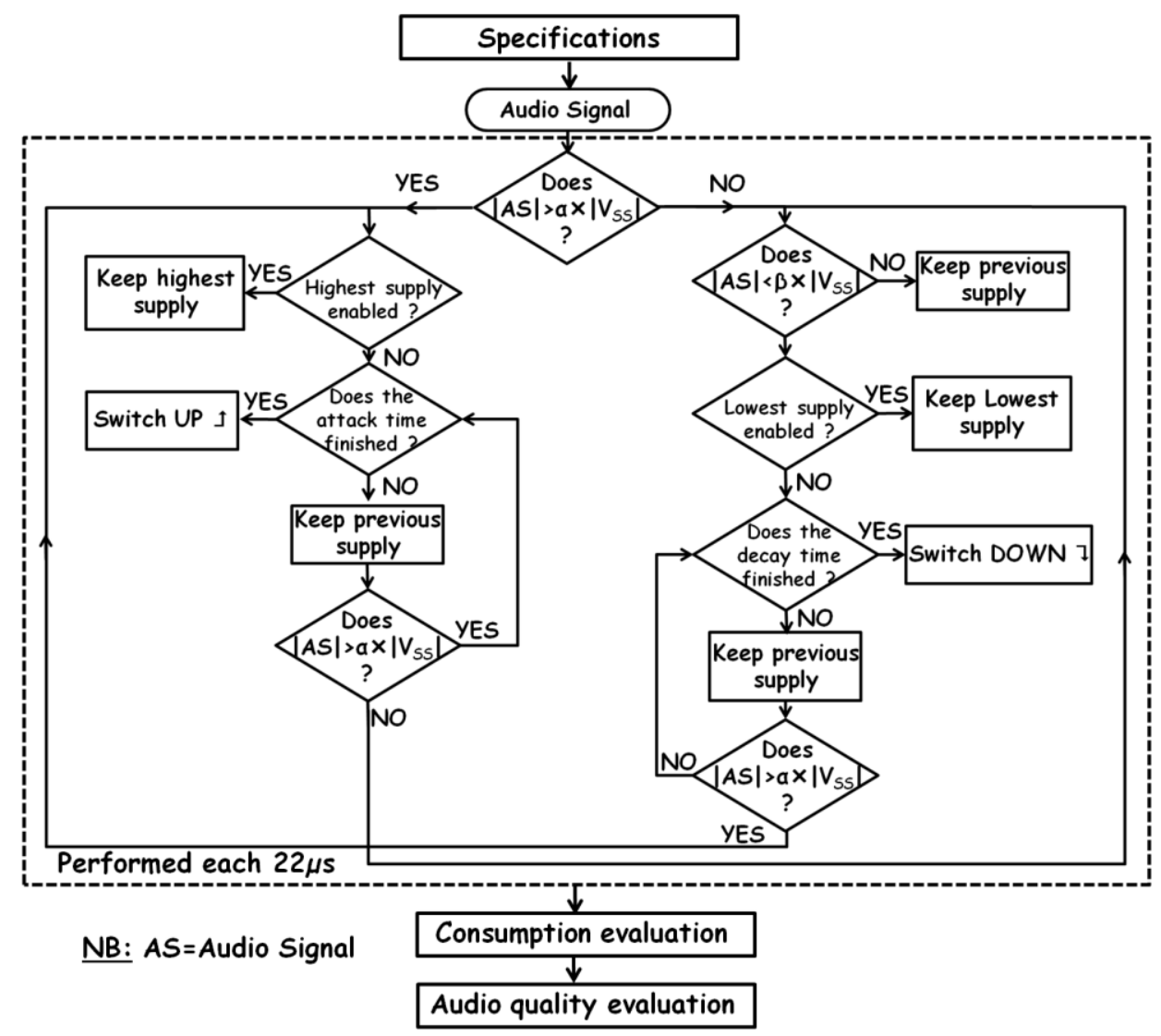

Fig. 5 Algorithm PSSA for two power supplies

To compare the efficiency of our PSSA, we introduce two continuous voltages tracking called real $\mathrm{H}$ and ideal H. At each calculation step, the power supply increase to maintain a constant gap between the audio signal and the power supply $\left(\mathrm{V}_{\mathrm{TRACK}}\right)$. Like the previous PSSA, the parameters $\alpha$ and $\beta$ fix the decision for the power supply rise and fall thresholds. The difference between the real and ideal $\mathrm{H}$ algorithms resides in the taking of this decision. In fact, as the study is being made for so-called «stand alone » circuits, the power supplies change one cycle after the comparators have detected the necessity to increase or reduce the power. We have called this type of amplifier real class-H in our modeling (see fig. 6). 


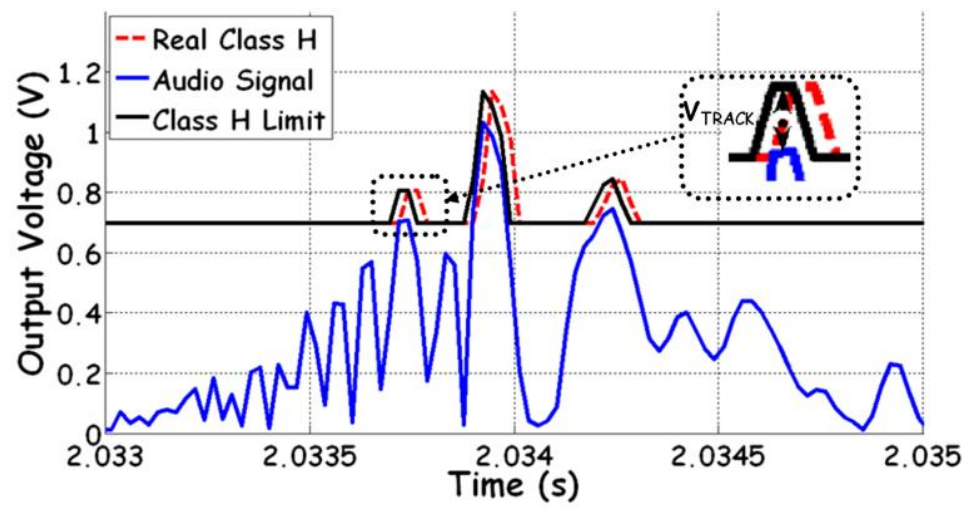

Fig. 6 Class-H principle

\subsubsection{Behavioral modeling of converters and linear amplifier}

Power supply switching can be done using a combination of linear and/switched, or capacitive and/inductive converter structures. In this work, the switching is done using a buck converter and a negative charge pump which is one of the most common configurations in industrial circuits [7]-[9].

The switching behavior of the two converters is the principal contributor to the long calculation time for the hybrid amplifier series. This behavior is also not very useful for predicting audio quality or power consumption. An average behavior was therefore chosen to reduce the calculation time for our model. The parameters modeling the converters are their efficiencies, quiescent current, rise time and output impedance. For example, the latter for the charge pump is defined by its switching frequency, and the parasitic capacitor and resistor values of the switches.

With the standard practice of using DC/DC converters on mobile phone platforms, the solution integrating two capacitive switch converters presently holds the attention of designers. In this case, only the parameters modeling the convertors need to be redefined. The implementation of the different PSSA does not put into question our modeling approach. In order to study only the influence of the PSSA, the linear amplifier is modeled with a fixed gain. Its linearity, noise level and power supply rejection are all considered as ideal. In the same way, the DC/DC converter is considered as having an ideal transient line and an ideal transient load but with an efficiency of $80 \%$ corresponding to DC/DC converters currently present in this type of circuit [7]. Finally, the negative charge pump is also considered as ideal (no $\mathrm{R}_{\mathrm{ON}}$ losses in the switches) but its equivalent resistor is modeled $\left(\mathrm{R}_{\mathrm{EQ}}=5 \Omega\right)$, as it is a contributor to the clipping of the signal in transient analysis. 


\subsubsection{Consumption evaluation}

Using the average behavior of the converters and modeling the amplifier enable an increase in the number of calculation points. Only the functioning frequency of the PSSA fixes the minimum number of calculation points. The power consumption of the hybrid amplifier series in stereo can be expressed at each calculation point by Eq. 2 :

$$
I_{B A T}=\frac{V_{D D}}{\eta_{B U C K} \times V_{B A T}}\left(I_{Q_{-} B U C K}+2 I_{L O A D}\right)+I_{Q_{-} B A T}
$$

where $\mathrm{I}_{\mathrm{BAT}}$ is the current consumed by the circuit, $\mathrm{I}_{\mathrm{Q} \_ \text {BUCK }}$ the quiescent current of the circuits fed by the $\mathrm{DC} / \mathrm{DC}$ converter, $\eta_{\mathrm{BUCK}}$ the efficiency of the DC/DC converter, $\mathrm{I}_{\mathrm{QBAT}}$ the quiescent current of the circuits connected directly to the battery and $\mathrm{I}_{\mathrm{LOAD}}$ the output current $\left(\mathrm{V}_{\text {OUT }} / \mathrm{R}_{\mathrm{LOAD}}\right)$, where $\mathrm{R}_{\mathrm{LOAD}}$ is the charge value considered as purely resistive.

The main advantage of this model is the reduction in the calculation time (cf. Table 1) by an averaged and simplified behavioral model, and an increase in the calculation point. This model therefore enables the simulation of signals over a longer period, as for real audio signals, in order to get closer to the real conditions. Optimization based on simulation also becomes possible in a reasonable time.

Table 1 Time Comparison

\begin{tabular}{ccc}
\hline Simulator & Signal Length & Simulation time \\
\hline Eldo & $10 \mathrm{sec}$ & 2.5 months (estimated) \\
Matlab & $10 \mathrm{sec}$ & $20 \mathrm{sec}$ \\
\hline
\end{tabular}

\subsection{Choice of input signal}

In the nominal conditions of use, the amplifier receives a complex audio signal where the amplitude and harmonic content vary significantly over time. A simulation time of several seconds is necessary to predict the behavior of the amplifier. In order to reduce this calculation time, a sinusoidal signal is generally used to simulate power consumption and audio quality. However, this type of signal does not represent the real conditions, notably because it falsifies the PSSA design and optimization. The non-periodicity and the crest factor of the audio signal provoke a switching of the power supply different to that simulated with a sinusoidal signal. 
Table 2 Signal used for test

\begin{tabular}{ccccc}
\hline Signal & Type & CF(dB) & Artist & Length(s) \\
\hline$\# 1$ & Sine Wave $(\mathrm{f}=1 \mathrm{kHz})$ & 3 & - & $50 \mathrm{~m}$ \\
\#2 & Audio & 14 & Janis Joplin & 10 \\
\#3 & Audio & 7 & David Guetta & 10 \\
\hline
\end{tabular}

This study has thus been done using two audio signals as well as the classical sinus pattern; the parameters are summarized in Table 2. The two audio signals are two styles of music (jazz and techno) with two different crest factors, thus representing two real cases of use. The frequency content of signal \#2 and the statistical occurrence for the three signals (for 15 classes and with absolute values) are shown on figs. 7 and 8 , respectively.

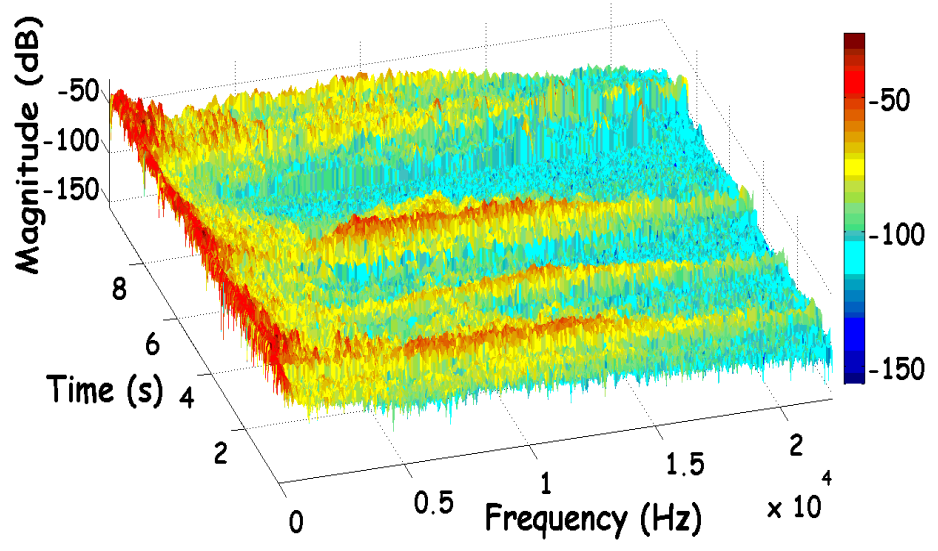

Fig. 7 Spectrogram of signal \#2 over 10s

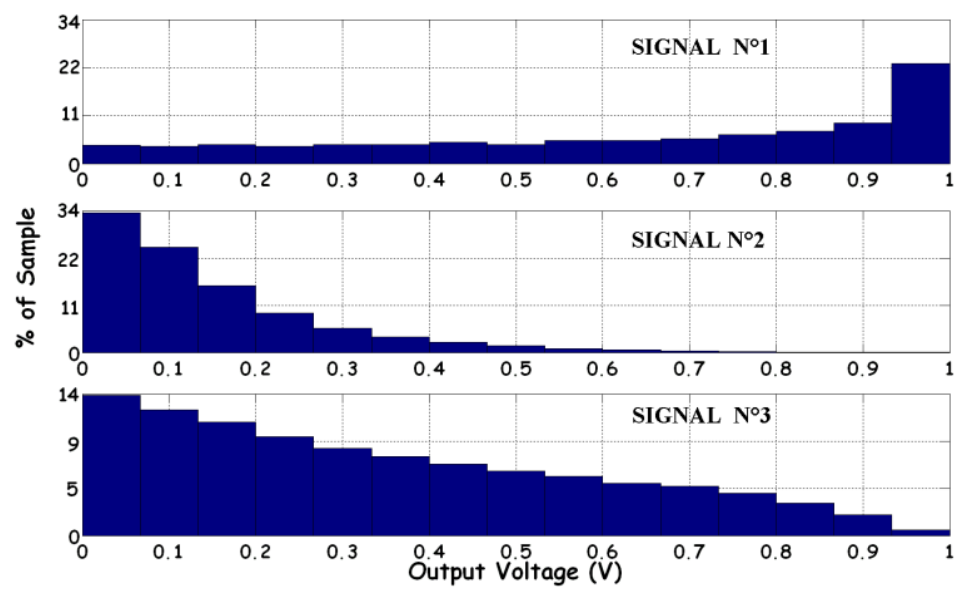

Fig. 8 Statistical occurrence for 3 test signals

\subsection{Required output power}

As the target of this paper is headphone applications, we done measurements of output power for such an application. In order to obtain a good audio level (75dB SPL), the output electrical power need to be lower 
than the maximum achievable power. In fact, as shown in fig. 9, audio tests on a Head and Torso simulator (Bruel \& Kjaer 4128C) were done. These were realized in an anechoic chamber, using two kind of headphone: a Sennheiser headphone (HD-595 with $50 \Omega$ impedance) which offer good performance and a cheap headphone (G-SOUND AP-310VR with $32 \Omega$ impedance), to see the leverage of their electroacoustical efficiency. We used a Rohde \& Schwarz UPD audio analyzer to perform the different tests. The tests were realized with a sinusoidal signal of $1 \mathrm{kHz}$ over a power range from $1 \mu \mathrm{W}$ to $20 \mathrm{~mW}$.
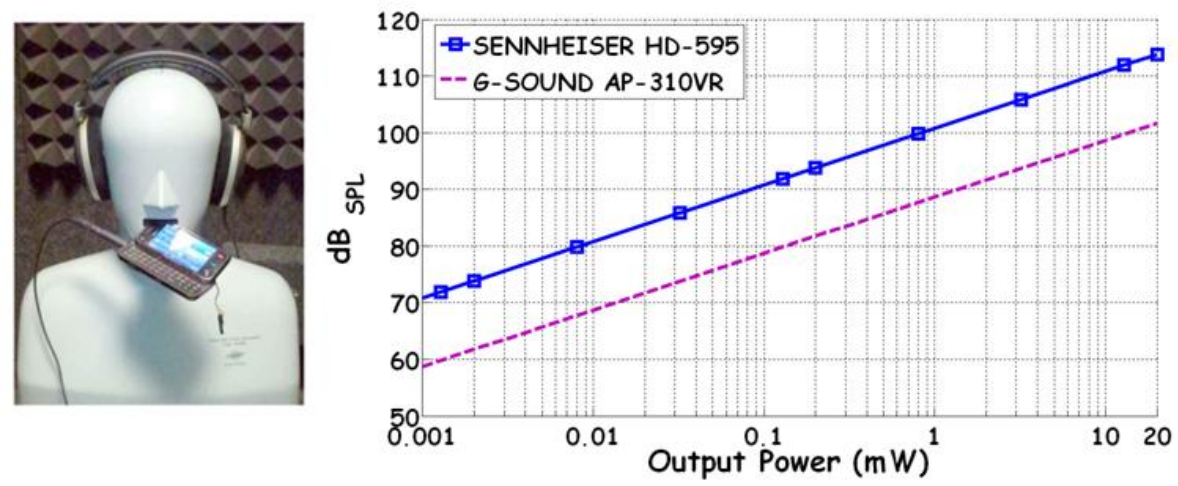

Fig. 9 Power needed in cell phone applications

The results showed that an acoustic power of $75 \mathrm{~dB}$ SPL is obtained for $3 \mu \mathrm{W}$ for the Sennheiser and $50 \mu \mathrm{W}$ for the G-sound, so very much less than the maximum output power fixed by industry [7]-[9]. However, as this study was done without any background noise, we have chosen to work with a higher power but one which is still relatively low i.e. $100 \mu, 500 \mu$ and $1 \mathrm{~mW}$, corresponding to 78,85 and $88 \mathrm{~dB}$ SPL (for the GSound), respectively, while privileging the lowest power during the optimizations.

\subsection{Evaluation of the audio quality}

Using multi-frequency and non-periodic audio signals prevents any classical frequency analysis such as the harmonic distortion (THD) (widely used to evaluate the audio quality). The association of three methods is thus suggested in order to evaluate the audio quality of our simulation results.

The first method uses the PEAQ (Perceptual Evaluation of Audio Quality) model which enables the audio quality of the output signal to be compared objectively to a reference signal using psycho-acoustic models [18]. This method gives a result, between 0 (imperceptible) and -4 (annoying), reflecting the music degradation perceived by the ears. The second method consists in doing comparative listening tests. This subjective evaluation is only useful to verify the first method. The third method completes our evaluation by measuring the time for the output signal to be clipped. 


\section{Optimization procedure}

The switching algorithm optimization approach is presented in this section. The objective is to find the algorithm parameters accurately and rapidly in order to minimize consumption over a wide power range, without degrading the audio quality. Two factors deserve particular attention to resolve an optimization problem, i.e. to know how to formulate the problem and the search algorithm.

\subsection{Formulation of the problem}

The formulation of the optimization problem consists in defining the search space of the variables, writing the cost function and defining the constraints that need to be respected.

\subsubsection{Search space of the variables}

The search space of the variables represents all combinations of the parameters of the PSSA.

Table 3 Design variables for each PSSA

\begin{tabular}{|c|c|c|c|c|c|}
\hline & 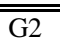 & G3 & G4 & Real H & Ideal H \\
\hline $\mathrm{V}_{\mathrm{DD} 1}$ & $\square$ & 口 & 可 & 可 & $\bar{\nabla}$ \\
\hline $\mathrm{V}_{\mathrm{DD} 2}$ & & 甲 & 口 & & \\
\hline $\mathrm{V}_{\mathrm{DD} 3}$ & & & $\square$ & & \\
\hline \multicolumn{6}{|l|}{$\mathrm{V}_{\mathrm{DD} 4}$} \\
\hline $\mathrm{V}_{\text {TRACK }}$ & & & & 曰 & 曰 \\
\hline$\alpha \& \beta$ & 甲 & 甲 & ఐ & 曰 & 甲 \\
\hline Decay Time & 口 & 口 & च & & \\
\hline Attack Time & 口 & 口 & च & च & 甲 \\
\hline Blanking Time & & 甲 & छ & & \\
\hline
\end{tabular}

Table 3 summarizes the optimization variables as a function of the switching algorithm studied $(\mathrm{G} n, \mathrm{H})$. It is important to point out that the maximum value of the power supply is fixed in order to limit the output power, imposed by legislation.

\subsubsection{Cost function}

The cost function is the unique mathematical expression of the performance of the system studied. The amplifier performance is defined by the power consumption as a function of the power delivered. It therefore consists of a multi-objective problem. To define a unique cost function, the linear aggregation approach was chosen [19]. This method, commonly used in the domain of analog synthesis [20], consists in transforming the multi-objective problem into a single objective one. This means adding all the objectives 
by attributing to each of them a weight coefficient representing a preference. The cost function is then written as follows:

$$
f=\sum_{i=1}^{3} \text { wi } \times \text { Ii with wi>0 and } \sum_{i=1}^{3} w i=1
$$

where, Ii is the consumption at each power, $\mathrm{i} \in[1,3]$ the number of the objective and wi the weighting coefficient.

In order to reduce the number of simulations, three significant power values were chosen: $100 \mu, 500 \mu$ and $1 \mathrm{~mW}$ as seen previously. These represent the real range used for listening. Their weighting coefficients are 0.6, 0.2 and 0.2, respectively. The overall evaluation of the performance will therefore give a greater weighting to the performance obtained at low power.

\subsubsection{Constraints}

The constraints are defined by the specifications respected during the optimization. In our study, the result of the PEAQ method must be greater than -0.5 to guarantee sufficient audio quality. Simulations giving results lower than this were not kept.

\subsection{Optimization algorithm}

Choosing an optimization algorithm leads to a reduction in the number of simulations done in order to find the optimal solution. The genetic algorithm (GA) [21] is one of the most, robust, rapid and popular algorithms for finding a quasi-optimal solution. It also does not need an initial solution because it is based on a random search method. However, it is slow and not very efficient for finding an optimal solution. On the contrary, the Pattern Search (PS) algorithm has the capacity to find the optimal solution if it starts with a good initial solution. Therefore, to optimize the switching algorithm, we sequentially combine the two optimization algorithms GA and PS. First, the GA searches over the whole search space of the variables for a quasi-optimal solution. Then, using this solution, the PS is used to locally affine the search to find an optimal solution.

\subsection{Optimization based on cascade simulations}

In order to evaluate the cost function, three simulations are done in parallel (fig. 10) using our previous models. This evaluation triples the number of simulations, but it allows an optimization over a wide power range: the choice of efficient optimization algorithm still being determined by the number of simulations to be done. 


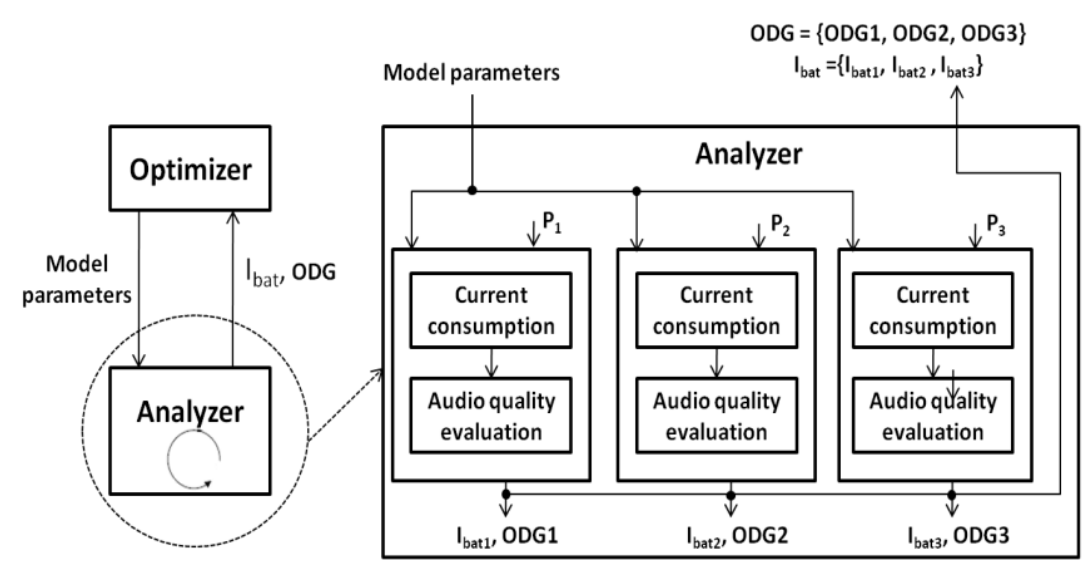

Fig. 10 Cascade simulation-based optimization

\section{RESULTS}

\subsection{Validation of our model}

Two class-G2 amplifiers [7]-[9] were used to validate our model. From the measurements, the input parameters of the model were found. The results by simulation were then compared to the characterization of these circuits at different power. Fig. 11 shows the test bench and the result of the comparison for circuit [7]. The configuration used here is a purely resistive charge of $47 \Omega$, a supply voltage of $3.6 \mathrm{~V}$ and different audio test signals. The relative error in the consumption is less than $5 \%$ over the whole power range tested for [7], and less than $10 \%$ for [8]. These results therefore validate the reliability of our modeling.
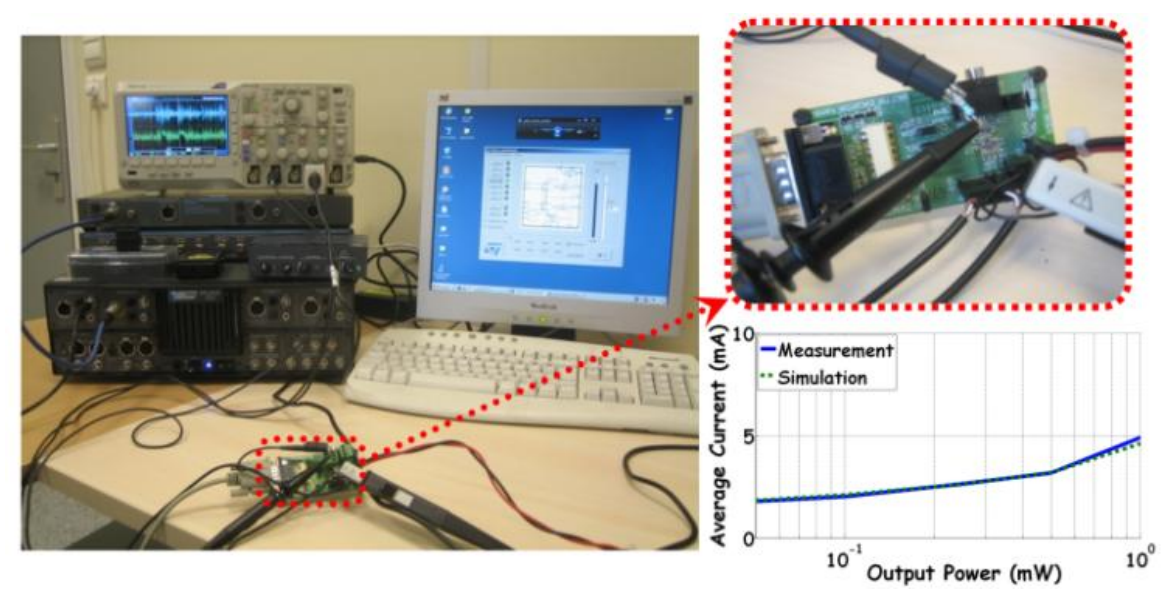

Fig. 11 Model/measurement comparison

\subsection{Influence of the signal on the power supply switching}

In fig. 12, we compare the consumption given by [7], simulated from the model, for the three test signals. The consumption is therefore linked to the signal used and particularly to the chosen PSSA. For example, 
the increase in power of $10 \mathrm{~mW}$ for signal \#1 corresponds to reaching the $\left|\alpha \times \mathrm{V}_{\mathrm{SS}}\right|$ threshold, thus switching the power supply to its second value. For the audio signals (\#2 and \#3), the rupture is more progressive when the crest factor is increased. The PSSA therefore has an influence at a power lower than that observed on signal \#1.

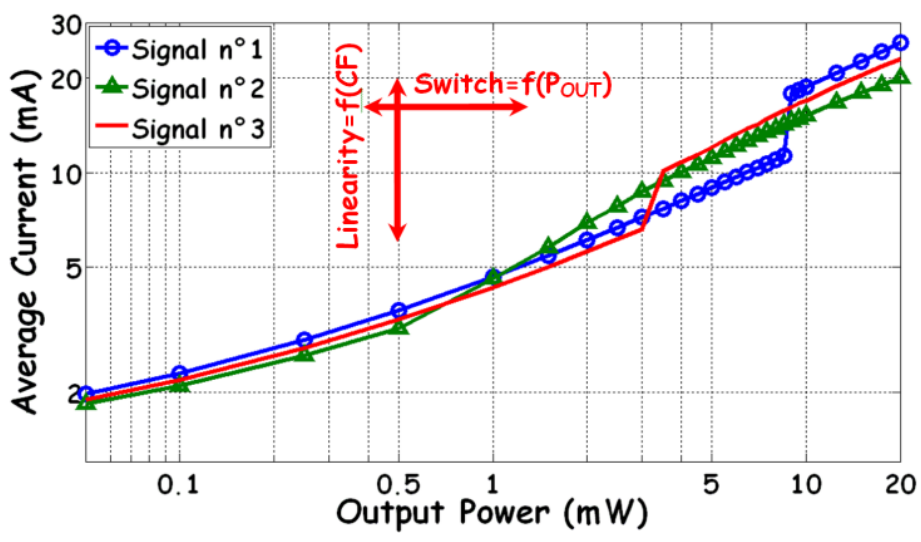

Fig. 12 Class-G2 consumption for the three test signals

\subsection{Parametric study}

Before proceeding to the optimization, the influence of each parameter of the PSSA is highlighted by varying one of them using the configuration given in [7]. In this paper, when nothing else is defined, we present the results for a nominal power of $1 \mathrm{~mW}$ and for signal \#2, and the audio quality (ODG) is $\geq-0.5$ which means that the audio quality has not been degraded: the crosses represent the state of the actual configuration of the circuit [7].

\subsubsection{Effect of the minimal power supply voltage}

The minimal power supply voltage is an important parameter in class-G amplifiers. It must be positioned such that it covers the whole dynamic range of the signal and is thus able to adapt to all types of audio signal. In the class-G2 amplifiers, only the lower power supply can be modified, as the high power supply is maintained at $1.9 \mathrm{~V}$ in order to ensure that a sufficient dynamic is obtained at the output $(\approx 20 \mathrm{~mW}$ with a sinusoidal at $3 \mathrm{~dB}$ ). The low power supply limit of these class-G amplifiers is set at $700 \mathrm{mV}$ even though the low voltage amplifiers $(450 \mathrm{mV})$ are presently being tested. Fig. 13 shows that a consumption gain can be obtained by modifying the power supply switching threshold with respect to the actual configuration. 


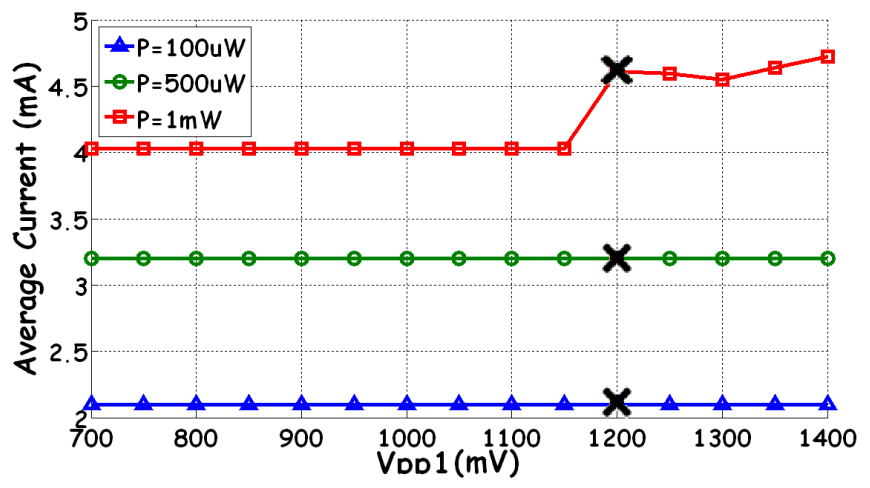

Fig. 13 The $V_{D D 1}$ effect

\subsubsection{Effect of the threshold voltage}

Fig. 14 shows the effect of the $\alpha$ and $\beta$ threshold voltages on the consumption. When the values of the threshold voltages are chosen as close as possible to the power supply, the consumption is reduced but the risk of signal clipping is high. In fact, it frequently occurs that the input signal value varies greatly, thus leaving insufficient time to establish the higher power supply value. In the present configuration in [7], the audio quality is not degraded. However, this problem has been highlighted during the different optimizations.

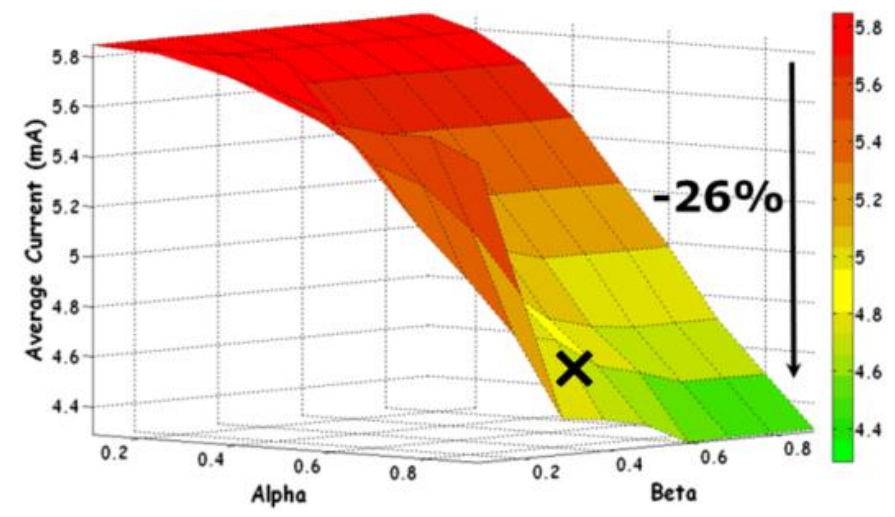

Fig. $14 \alpha \& \beta$ effects on current consumption

\subsubsection{Effect of decay time}

The decay time of the higher power supply value is a determining factor on the consumption. Fig. 15 shows the effect of decay time on the consumption and audio quality when using signal $\# 2$. The decrease of the decay time with respect to the initial configuration in [7] enables the consumption to be decreased by $13 \%$ without degrading the audio quality. 


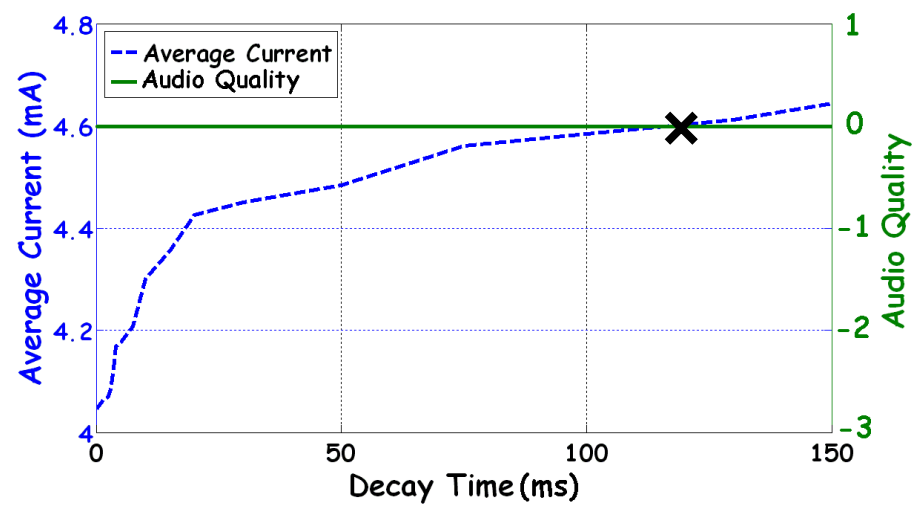

Fig. 15 Decay time effect

\subsubsection{Effect of attack time}

The variation of the attack time shows us that this parameter can be useful for signals with a high crest factor $(\mathrm{CF})$, but degrades the audio quality too much for a low CF. Fig. 16 shows the response of the audio quality for signal \#3, which has a low CF. With this signal the PEAQ method gives a value lower than -0.5 which means that the audio quality is deteriorate.

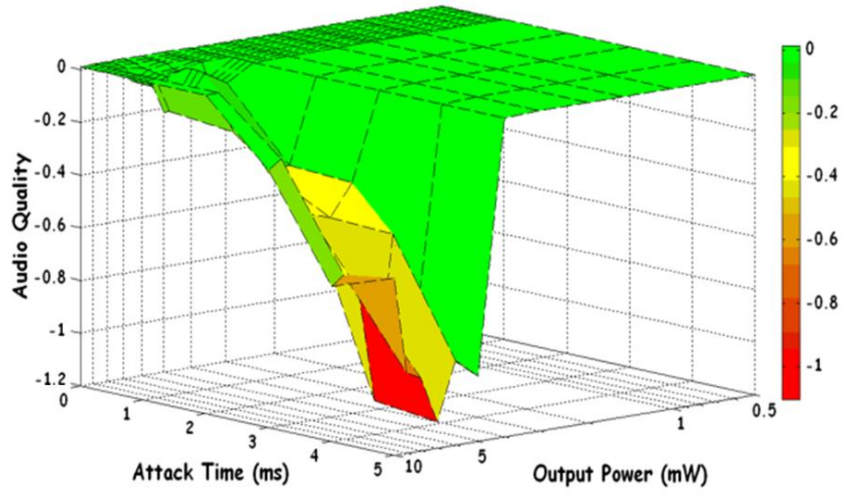

Fig. 16 Attack time effect for low crest factors

In order to find the best trade off in PSSA parameters, the next sections propose an overall optimization.

\subsection{Comparison of the optimization algorithms}

Two different algorithms, GA-SQP and GA-PS were compared for optimizing [7] (type G2). GA-SQP is a hybrid algorithm based on the global algorithm GA, associated with a local search algorithm called the Sequential Quadratic Programming (SQP) algorithm. The optimization toolbox in Matlab was used to implement the optimizations. Table 4 compares the minimal consumption found by each algorithm as well as the number or iterations to find this solution. As can be seen, the two coupled algorithm allow minimizing the consumption within a reasonable time. The GA-PS offer slightly better results. This will therefore be used to optimize the PSSA. 
Table 4 Algorithm comparison

\begin{tabular}{ccc}
\hline & Hybrid GA-SQP & Hybrid GA-PS \\
\hline Number of Iterations & 576 & 554 \\
Cost function (mA) & 2.6 & 2.41 \\
Simulation Time (min) & 47 & 45 \\
Results & $\oplus$ & - \\
\hline
\end{tabular}

Without an optimization algorithm, more than 100000 simulations would be necessary for an exhaustive search. Furthermore, using our proposed model enables a reduction in the optimization time by at least one hour. By comparison, 134 years of calculation time would have been necessary if the models based on only the electronics had been used on an electrical level.

\subsection{Optimization of the PSSA of circuit [6]}

The suggested optimization enables us, without a starting point (no expertise is needed), to automatically find the best compromise between the values of all the parameters (Table 3) to minimize the consumption without degrading the sound reproduction quality. The GA-PS optimization was applied to the class-G2 amplifier [7] in order to reduce its consumption while at the same time respecting the electrical constraints $\left(\mathrm{V}_{\mathrm{DD} 1}>0.7 \mathrm{~V}\right)$. Fig. 17 compares the simulated consumption of [7] and the optimized PSSA with signal \#2. The optimization enables a reduction in consumption from 18 to $30 \%$ over the whole power range.

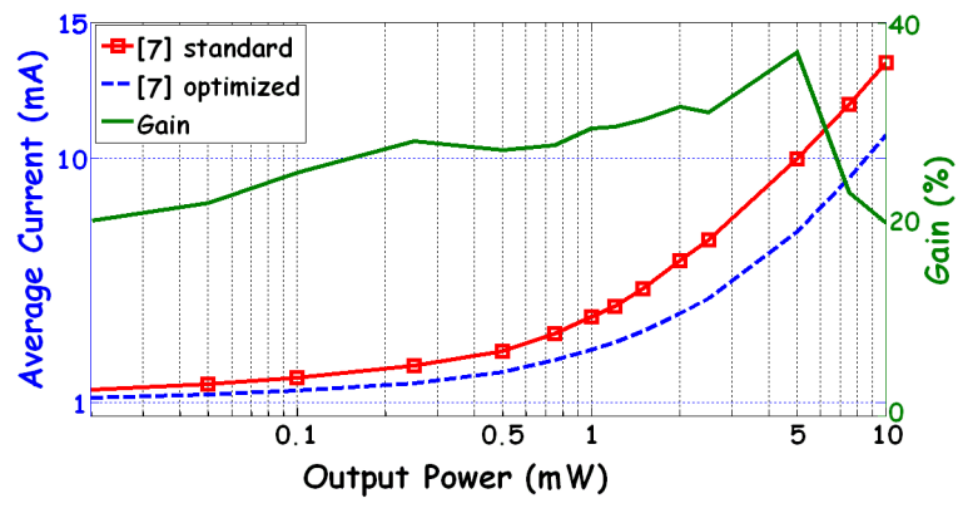

Fig. 17 Comparison [datasheet]/optimized circuit for signal \#2

To prove the reliability of our optimization we used a signal not used during the different process optimizations. This signal, lasting 10 seconds, possesses a CF of $16 \mathrm{~dB}$ and is interpreted by the Red Hot Chili Peppers. It will be called signal \#4. Fig. 18 shows the comparison of the results of [7] in standard configuration and [7] optimized. 


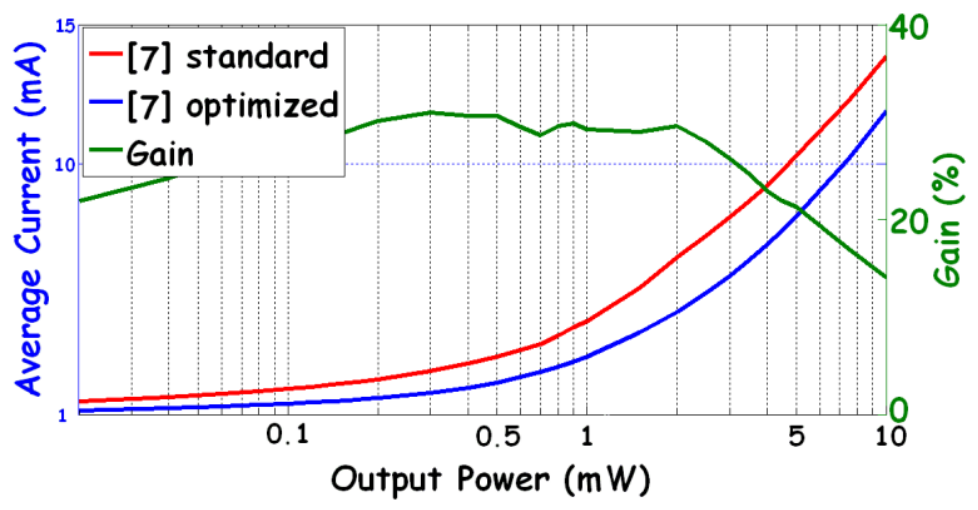

Fig. 18 Comparison [datasheet]/optimized circuit for signal \#4

We can see that the gain in consumption from $20 \mu \mathrm{W}$ until $2 \mathrm{~mW}$ is between 20 and $35 \%$. This gain in consumption is nearly the same that the audio signals which were used for optimization. It means that our optimization is robust and the audio test signals used represents a major use of cases. Three others test signals have been simulated and leads to the same conclusion.

\subsection{Comparison of the different PSSA strategies}

In order to reduce the consumption, the different PSSA strategies are studied. Figs. 19 and 20 compare the consumption obtained from the different optimized strategies for signals \#1 and \#2. All the optimizations respect the audio quality constraint, defined previously, and a minimal supply voltage fixed at $700 \mathrm{mV}$.

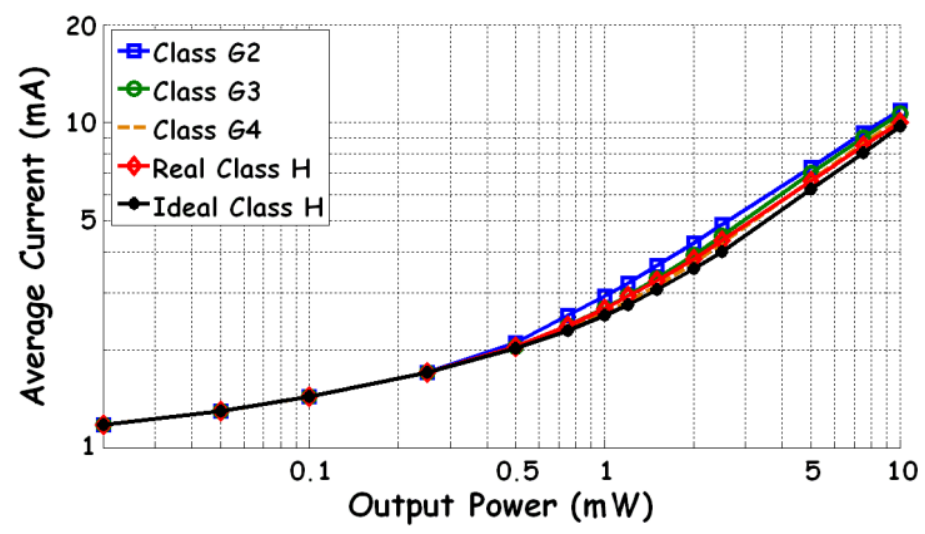

Fig. 19 Signal \#2 @ 0.7V

As we can see in fig. 19, in nominal conditions (power less than $1 \mathrm{~mW}$ ), the introduction of one or two supplementary power supply values reduces the consumption when an audio signal is used, but once $500 \mu \mathrm{W}$ of output power is reached. Analysis using a sinusoidal signal (fig. 20) leads to quite a different conclusion, as switching is seen at higher power. 


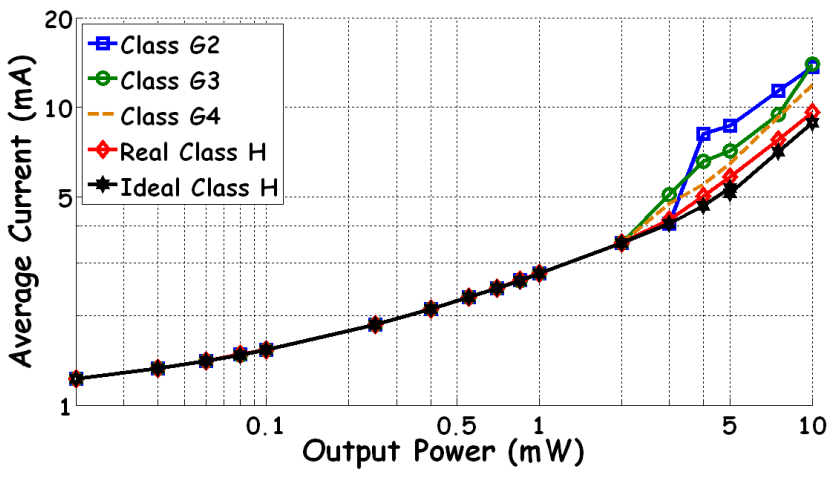

Fig. 20 Signal \#1 @0.7V

\subsection{Influence of the minimal power supply voltage}

The minimal power supply voltage associated with the PSSA strategy also influences the consumption for an output power less than $500 \mu \mathrm{W}$. Figs. 21 and 22 compare the consumption for different optimized strategies with a minimal power supply voltage of $450 \mathrm{mV}$. This voltage was voluntarily chosen because some amplifiers presently being tested allow the voltage to be lowered to $450 \mathrm{mV}$. The highest supply voltage is always fixed at $1.9 \mathrm{~V}$ to respect the maximal power delivered at the output $(\approx 20 \mathrm{~mW}$ for a sinusoidal at $3 \mathrm{~dB})$.

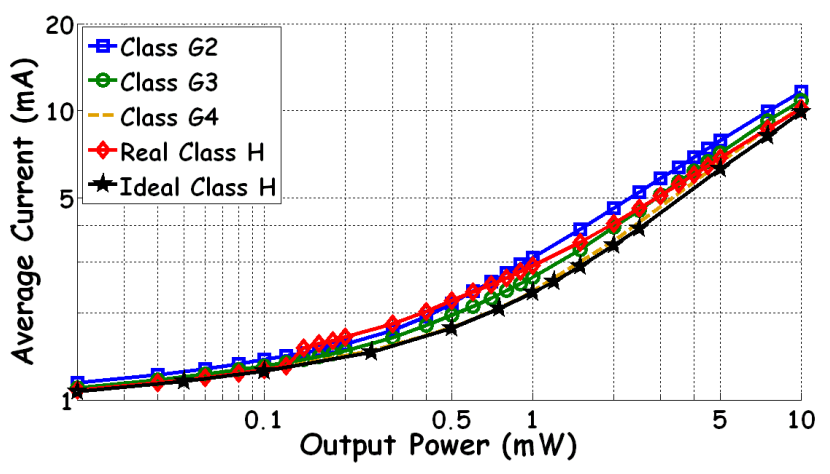

Fig. 21 Signal \#2 @ 450mV

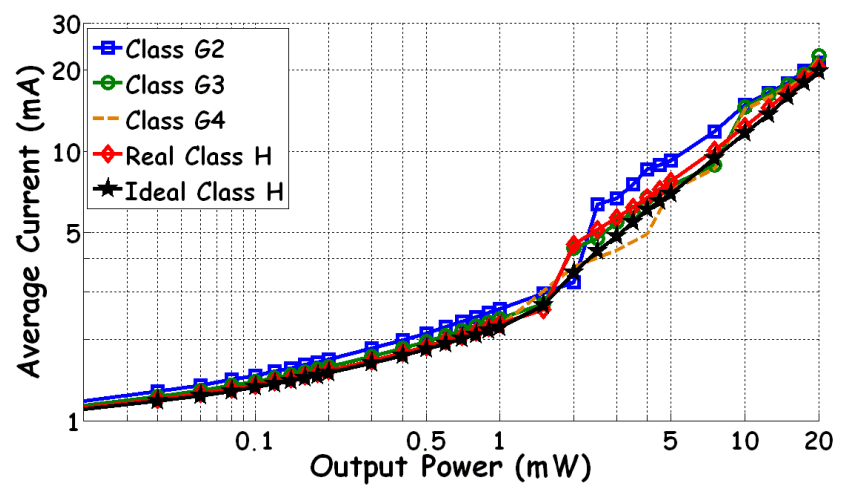

Fig.22 Signal \#1 @ 450mV 
As we can see from figs. 21 and 22, the introduction of two supplementary voltages (G4) significantly reduces the consumption over the nominal functioning range $(100 \mu \mathrm{W}$ to $1 \mathrm{~mW})$ with respect to the $\mathrm{G} 2$ and G3 strategies. For example, with signal \#2, 9\% in gain of consumption is obtained at $100 \mu \mathrm{W}$ for the ClassG4 compared to the Class-G2 optimized and $24 \%$ for an output power of $1 \mathrm{~mW}$. The choice of PSSA strategy is therefore different from the previous paragraph. A low minimal voltage requires a multiplication of the intermediate values to efficiently manage the whole of the dynamic of the signal.

Table 5 includes all the value of the parameters optimized for a minimal supply voltage of $450 \mathrm{mV}$. We can see that the trend of $\mathrm{V}_{\mathrm{DD}}$ is to decrease with the add of a power supply, leading the minimal supply $\mathrm{V}_{\mathrm{DD}}$ to get closer to $0.45 \mathrm{~V}$. The decay time follow the same trend and the ratio of the supply ( $\alpha$ and $\beta$ ) are almost the same for each PSSA topology. From Table 5 and fig. 21, we can understand why the PSSA of Class-G4 is better than a real Class-H. The track supply ( $\left.\mathrm{V}_{\text {TRACK }}\right)$ is very important $(625 \mathrm{mV})$ to avoid clipping of the audio signal. The audio signal (especially with a high $\mathrm{CF}$ ) evolves very quickly and thus tracking permanently the audio signal is not the best PSSA strategy. The Class-G4 strategy is the most efficient voltage tracking and allows an easier implementation in transistor level.

Table 5 Value of the optimized parameters

\begin{tabular}{|c|c|c|c|c|c|}
\hline & G2 & G3 & G4 & Real H & Ideal $\mathrm{H}$ \\
\hline $\mathrm{V}_{\mathrm{DD} 1}(\mathrm{~V})$ & 0.64 & 0.57 & 0.52 & 0.53 & 0.51 \\
\hline $\mathrm{V}_{\mathrm{DD} 2}(\mathrm{~V})$ & 1.9 & 1.26 & 0.89 & -- & -- \\
\hline $\mathrm{V}_{\mathrm{DD} 4}$ or $\mathrm{V}_{\mathrm{MAX}}(\mathrm{V})$ & -- & -- & 1.9 & 1.9 & 1.9 \\
\hline $\mathrm{V}_{\text {TRACK }}(\mathrm{mV})$ & -- & -- & -- & 625 & 570 \\
\hline Decay Time $(\mu \mathrm{s})$ & 350 & 260 & 180 & -- & -- \\
\hline Attack Time (s) & 0 & 0 & 0 & 0 & 0 \\
\hline Blanking Time $(\mu \mathrm{s})$ & -- & 50 & 50 & -- & -- \\
\hline
\end{tabular}

We present in fig. 23 the efficiency defined by $\mathrm{P}_{\mathrm{OUT}} / \mathrm{P}_{\mathrm{IN}}$ of all the PSSA strategy for the same optimized parameters than those presented in Table 5 but for a sample of music not used during the optimization process like we do previously to compared the actual configuration of Class-G2 and its optimized version. The signal \#4 was chosen, and the results lead to the same conclusion, namely that the PSSA strategy of Class-G4 is the best strategy. To highlight the need of increasing the number of supply and to chose correctly the value of the different parameters of the PSSA, fig. 24 presents the gain in efficiency obtained in absolute value by taking the class-G2 optimized as reference. We can see (in absolute value) that $10 \%$ of efficiency at $100 \mu \mathrm{W}$ and $27 \%$ of efficiency can be obtained with the Class-G4 strategy compared to the Class-G2 optimized strategy. On the contrary, the real Class-H amplifier is less efficient than the Class-G2 
amplifier for low power, emphasizing once again the fact that this strategy is not interesting in our application.

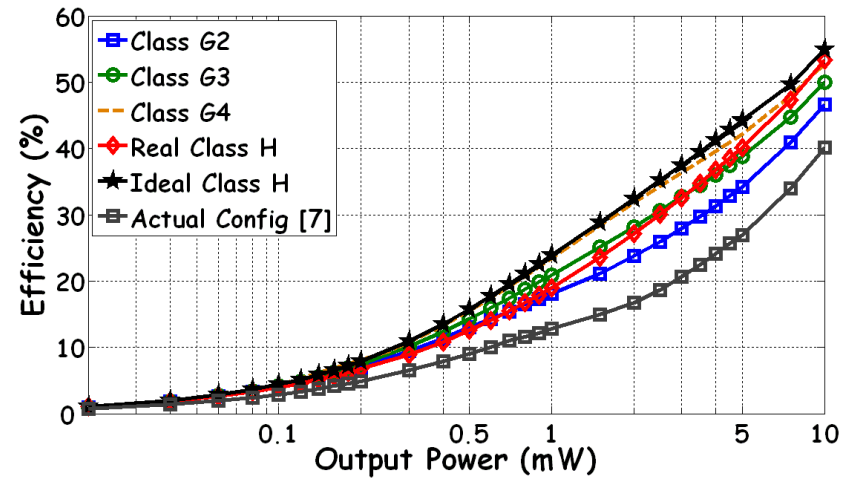

Fig. 23 Efficiency for signal \#4

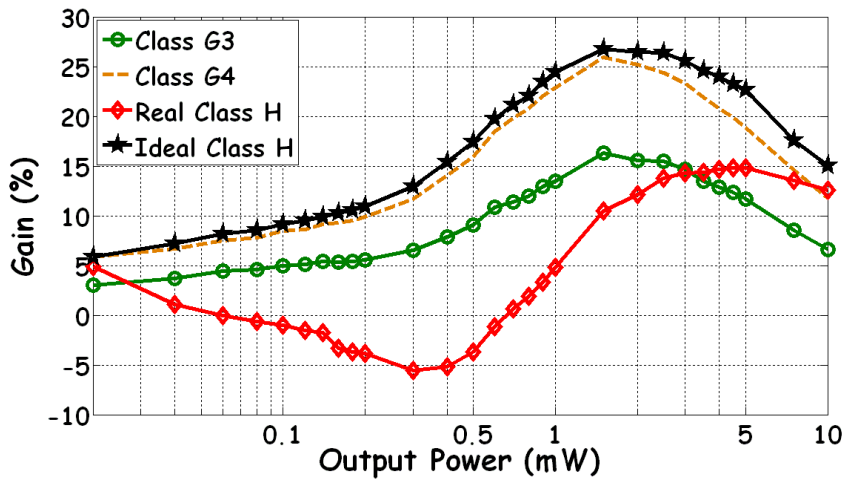

Fig. 24 Efficiency gain for signal \#4

\section{Conclusion}

To conclude, this article has presented a study of the PSSA strategy for a series hybrid amplifier dedicated to headphones in order to reduce its power consumption. First, a behavioral modeling was done to simulate the consumption and predict the audio quality as a function of a reconfigurable PSSA. This modeling enabled a significant reduction in calculation time, thus allowing the simulation of realistic signals of several seconds. The model has also been validated on two existing circuits. Furthermore, it could be easily adapted to other electrical architectures.

Second, an optimization approach based on the simulation of the model has been presented. The GA-PS hybrid algorithm allowed us to optimize and compare different PSSA strategies. The power range and nature of the input signal were taken into account to ensure that the optimizations were valid for nominal conditions of use. The introduction of four power supply values enabled us to get close to the optimal power consumption of the class- $\mathrm{H}$ amplifier, thus avoiding an increase in the PSSA complexity, since only 
few logic gates have to be added into the level detector compared to current solution. The reduction in the minimal voltage also highlights the interest of introducing several power supply voltages. In this case, the power consumption is reduced over a wide power range at the same time respecting the audio quality and a wide dynamic range at the output.

\section{References}

[1] Ben Duncan, High Performance Audio Power Amplifier, Newnes, 1997.

[2] M. Mijic, D. Masovic, D. Sumarac Pavlovic and M. Petrovic, "Statistical Properties of Music Signals", Audio Engineering Society 126th Convention, 2009.

[3] G. Pillonnet, R. Cellier, E. Allier, N. Abouchi, and A. Nagari, "A topological comparison of PWM and hysteresis controls in switching audio amplifier”, IEEE Asia Pacific Conference on Circuits and Systems, pp. 668-671, 2008.

[4] A. Nagari, "Tutorial review: audio amplifiers in mobile platforms", Analog Integrated Circuits and Signal Processing, Springer, pp 1-10, 2011.

[5] R.A.R Van der Zee and E.A.J.M Van Tujil, “A power-efficient audio amplifier combining switching and linear techniques”, IEEE Journal of Solid State Circuits, vol. 34, no. 17, pp. 995-991, 1999.

[6] E. Sturtzer, G. Pillonnet, A. Huffenus, N. Abouchi, F. Goutti and V. Rabary, "Improved Class-K Amplifier for Headset Applications", In 8th IEEE International NEWCAS Conference, pp. 185-188, 2010.

[7] TS4621 High performance class G stereo headphone amplifier with $\mathrm{I}^{2} \mathrm{C}$ volume control Datasheet, ST Microelectronics products, February 2010.

[8] TPA6140 Class-G DirectPath stereo headphone amplifier with $\mathrm{I}^{2} \mathrm{C}$ volume control Datasheet, Texas Instruments products, March 2009.

[9] LM48824 Class-G headphone amplifier with $\mathrm{I}^{2} \mathrm{C}$ volume control Datasheet, National Semiconductor products, August 2009.

[10] R. Bortoni, S.N. Filho and R. Seara, "Analysis, Design and Assessment of Class A, B, AB, G and H Audio Power Amplifier Output Stage Based on MATLAB ${ }^{\circledR}$ Software”, 110th Audio Engineering Society conferences, 2001.

[11] F. H. Raab, “Average Efficiency of Class-G Power Amplifier”, IEEE transaction on Consumer Electronics, vol. 32, no. 2, pp. 145-150, 1986.

[12] E. Mendenhall, "Computer Aided Design and Analysis of Class B and Class H Power Amplifier Output Stage", Audio Engineering Society 101st Convention, 1996.

[13] T. Sampei, S.Ohashi, Y. Ohta and S. Inoue, "Highest Efficiency and Super Quality Audio Amplifier Using MOS Power FETS in Class G Operation”, IEEE Transaction on Consumer Electronics, Vol. 24, no.3, 1978.

[14] A. Lollio, G. Bollati, R. Castello, “A class-G Headphone Amplifier in 65nm CMOS Technology”, IEEE Journal of Solid State Circuits. vol. 45, no.12, 2010.

[15] A. Downey and G. Wierzba, “A Class-G/FB Audio Amplifier”, IEEE Transactions on Consumer Electronics. vol.53, no. 4, 2007.

[16] J. Gubelmann, P.A. Dal Fabro, M. Pastre and M. Kayal, "High-efficiency dynamic supply CMOS audio power amplifier for low-power applications", Journal of Microelectronics, Elsevier, 2009.

[17] G. Pillonnet, M. Chiollaz, N. Abouchi and P. Marguery, "High Performance Simulator for Digital Audio class D Amplifiers", IET Transaction Circuits, Devices and Systems, 2010. 
[18] T. Thiede and al, "PEAQ The ITU Standard for Objective Measurement of Perceived Audio Quality", Journal of Audio Engineering Society, vol.48, no.1, pp. 3-29, 2000.

[19] N. Srinivas and K. Deb, "Multiobjective optimization using nondominated sorting in genetic algorithms", Journal of Evolutionary Computation, vol.2, pp. 221-248, 1994.

[20] R.M. Lewis and V. Torczon, "Pattern Search Algorithms for Bound Constrained Minimization", Journal SIAM on Optimization, vol. 9, 1999.

[21] E.G. Talbi, "Metaheuristics: From Design to Implementation", Ed. Wiley Series on Parallel and Distributed Computing, pp. 190-237, 2009. 